\title{
Bypassing adverse injection reactions to nanoparticles through shape modification and erythrocyte 'hitch-hiking'
}

Peter Pope Wibroe, ${ }^{1}$ Aaron C. Anselmo, ${ }^{2}$ Per H. Nilsson,,${ }^{3,4}$ Apoorva Sarode, ${ }^{2}$ Vivek Gupta, ${ }^{5}$ Rudolf Urbanics, ${ }^{6}$ Janos Szebeni, ${ }^{6}$ Alan Christy Hunter, ${ }^{7}$ Samir Mitragotri, ${ }^{2}$ Tom Eirik Mollnes, ${ }^{3,8,9}$ Seyed Moein Moghimi ${ }^{1,10,11^{*}}$

${ }^{1}$ Nanomedicine Laboratory, Centre for Pharmaceutical Nanotechnology and Nanotoxicology, Department of Pharmacy, Faculty of Health and Medical Sciences, University of Copenhagen, Universitetsparken 2, DK-2100 Copenhagen Ø, Denmark

${ }^{2}$ University of California at Santa Barbara, Department of Chemical Engineering and Center for Bioengineering, Santa Barbara, CA 93106, USA

${ }^{3}$ Department of Immunology, Oslo University Hospital Rikshospitalet, and K.G. Jebsen IRC, University of Oslo, 0372 Oslo, Norway

${ }^{4}$ Linnaeus Centre for Biomaterials Chemistry, Linnaeus University, 39182 Kalmar, Sweden

${ }^{5}$ College of Pharmacy and Health Sciences, St. John's University, 8000 Utopia Parkway, Queens, NY 11439, USA

${ }^{6}$ Nanomedicine Research and Education Center, Semmelweis University, Budapest \& SeroScience Ltd, Budapest, Hungary

${ }^{7}$ Leicester School of Pharmacy, De Montfort University, The Gateway, Leicester LE1 9BH, UK

${ }^{8}$ Reserach Laboratory, Nordland Hospital, 8092 Bodø, and K.G. Jebsen TREC, University of Tromsø, 9037 Tromsø, Norway

${ }^{9}$ Centre of Molecular Inflammation Research, Norwegian University of Science and Technology, 7491 Trondheim, Norway

${ }^{10}$ Nano-Science Center, University of Copenhagen, Universitetsparken 5, DK-2100 Copenhagen $\varnothing$, Denmark

${ }^{11}$ School of Medicine, Pharmacy and Health, Durham University, Queen's Campus, Stockton-on-Tees TS17 6BH, United Kingdom

*Corresponding author: School of Medicine, Pharmacy and Health, Durham University, Queen's Campus, Stockton-on-Tees TS17 6BH, United Kingdom

E.mails: moein.moghimi@gmail.com; seyed.m.moghimi@durham.ac.uk 

nanoparticles, induce adverse cardiopulmonary reactions in sensitive human subjects and these reactions are highly reproducible in pigs. While the underlying mechanisms are poorly understood, roles for both the complement system and reactive macrophages have been implicated. Here, we show the dominance and importance of robust pulmonary intravascular macrophage clearance of nanoparticles in mediating adverse cardiopulmonary distress in pigs and irrespective of complement activation. Specifically, we show that delaying particle recognition by macrophages within the first few minutes of injection overcomes adverse reactions in pigs using two independent approaches. First, we changed particle geometry from a spherical shape (which triggers cardiopulmonary distress) to either rod- or disk-shape morphology. Second, we physically adhered spheres to the surface of erythrocytes. These strategies, which are distinct from commonly leveraged stealth engineering approaches such as nanoparticle surface functionalization with poly(ethylene glycol) and/or immunological modulators, prevent robust macrophage recognition resulting in the reduction or mitigation of adverse cardiopulmonary distress associated with nanopharmaceutical administration.

Intravenous administration of liposomal and polymeric nanopharmaceuticals is

known to incite autonomic, muco-cutaneous and cardiopulmonary reactions in some

73 human patients. ${ }^{1-5}$ Symptoms include fever, chills, wheezing, facial swelling,

74 flushing, rash, coughing, shortness of breath, tachypnea, hypertension/hypotension

75 and chest and back pain. These symptoms range from mild to severe and are not

76 initiated by pre-existing allergen-reactive immunoglobulins (e.g., IgE type

77 antibodies). ${ }^{4,5}$ Despite this, current nanopharmaceuticals used in the clinic are not

78 designed to overcome these issues.

The underlying mechanism(s) behind intravenous injection reactions to nanopharmaceuticals is poorly understood. Inadvertent activation of the complement system, which is the first line of the body's defence against foreign intruders, has

82 been suggested to be a causal factor. ${ }^{4,5}$ Liberated complement anaphylatoxins C3a and

83 C5a can modulate the function of responder immune cells such as mast cells, 84 neutrophils, basophils, eosinophils and macrophages causing rapid release of 
85 secondary mediators that negatively affect the cardiovascular system. ${ }^{4,6,7}$

86 Nanopharmaceutical-mediated cardiopulmonary responses in sensitive human

87 subjects are highly reproducible in pigs, which include a massive increase in

88 pulmonary arterial pressure (PAP) and decline in the systemic arterial pressure

89 (SAP). ${ }^{8}$ Moreover, earlier studies have shown a role for complement activation and

90 particularly C5a in the development of cardiopulmonary distress in pigs. ${ }^{9}$

91 Unlike humans, pigs and sheep have resident pulmonary intravascular

92 macrophages (PIMs). ${ }^{10,11}$ PIMs instantaneously ingest intravenously injected particles

93 and subsequently release large quantities of thromboxane A2 (TxA2), prostaglandins

94 and prostacyclins that correlate with periods of peak vasoconstriction,

95 bronchoconstriction and pulmonary hypertension. ${ }^{11}$ Furthermore, earlier studies have

96 demonstrated that newborn lambs, prior to developing PIMs show no changes in PAP

97 after particle injection. ${ }^{12}$ Within two weeks of birth, lambs develop a population of

98 PIMs, which is accompanied by increased lung accumulation of injected particles

99 with a concomitant increase in PAP and TxA2 production. ${ }^{12}$ Collectively, these

100 observations suggest that PIMs, on robust phagocytosis may induce anaphylaxis.

101 Complement anaphylatoxins may further modulate the function of PIMs as well as

102 other immune cells and aggravate cardiopulmonary reactions. ${ }^{13}$ For instance, C5a can

103 synergistically enhance Toll-like receptor-induced production of pro-inflammatory

104 cytokines and further promote TxA2 release. ${ }^{14}$ In line with the role of macrophages in

105 anaphylaxis, a recent hypothesis has suggested that human subjects who are sensitive

106 to nanopharmaceutical administration presumably have a subset of highly responsive

107 resident macrophages in pulmonary circulation. ${ }^{15}$ Indeed, there are suggestions of

108 induction of pulmonary macrophages in subjects with liver abnormalities and other

109 hepato-pulmonary diseases..$^{10,11,16,17}$ 
111 internalization may present an attractive means to limit nanopharmaceutical-mediated

112 cardiopulmonary distress. Surface modification of nanoparticles with poly(ethylene

113 glycol) (PEG) is a well-established strategy to combat rapid macrophage

114 interception. ${ }^{18}$ Unfortunately, acute adverse injection reactions with PEGylated

115 liposomes and nanoparticles still persist in some human subjects as well as in 116 pigs. ${ }^{4,9,18-20}$ Indeed, PEGylated particles can not only trigger complement 117 activation. ${ }^{18-22}$ but they are also prone to rapid recognition by a subset of 118 monocyte/macrophage populations independent of opsonization processes. ${ }^{23,24}$

119 Alternative approaches are therefore necessary for preventing particle120 macrophage interaction within the first few minutes of injection where reactions 121 typically develop. Recently, it was shown that particle shape could be a pivotal 122 parameter in combating recognition by macrophages. ${ }^{25-28}$ Parallel to these attempts, 123 particle 'hitch-hiking' on erythrocytes also afford protection to robust particle 124 ingestion by macrophages in contact with the blood. ${ }^{29,30}$ Here, we employ these 125 strategies, which may be applicable to currently available spherically- and non-

126 spherically-shaped nanomedicines, ${ }^{31}$ and show that by leveraging both particle shape 127 modifications and erythrocyte 'hitch-hiking', adverse cardiopulmonary reactions 128 occurring due to nanoparticle bolus injection can be dampened or overcome. 129 Therefore, we demonstrate a transitional link from robust clearance of nanoparticles 130 by strategically placed macrophages (as in PIMs) in systemic circulation to adverse 131 haemodynamic reactions. 


\section{The effect of particle shape}

136 We utilized carboxylated polystyrene particles of spherical, prolate ellipsoidal (rods) 137 and oblate ellipsoidal (disks) shapes bearing a comparable range of Gaussian

138 curvatures (Fig. 1). We assessed propensity of these particles to 1) incite complement

139 in pig $^{32}$ and human blood ${ }^{21,22}$, and 2) induce haemodynamic disturbances in vivo in 140 the pig model. ${ }^{8,9}$

141 The results in Fig. 2a\&b show the effect of the particle shape on time142 dependent complement activation in the pig blood. Complement activation was 143 monitored through measurements of sC5b-9 (a nonlytic soluble marker of the terminal 144 pathway of the complement system and a sensitive measure of the activation of the 145 whole complement cascade) and anaphylatoxin $\mathrm{C} 5 \mathrm{a},{ }^{21,22,33}$ relative to a zymosan (an 146 established potent activator of the complement system) response. Absolute values of 147 complement activation products are given in Supplementary Fig. 1. We compared 148 complement activation at an equivalent surface area for each particle type $(\sim 14,500$ $149 \mathrm{~mm}^{2} / \mathrm{mL}$ of blood). Spheres $(500 \mathrm{~nm})$ did not incite complement within the first 5 $150 \mathrm{~min}$ of incubation. At later time-points complement activation was minor, but 151 statistically significant (Fig. 2a \& b). Similar to spheres, rods and disks did not induce 152 complement activation within the first $5 \mathrm{~min}$ of contact with blood, but later 153 complement activation was robust and more profound than spheres. Since stretching 154 spheres at high temperature generated rods and disks, these conditions may have 155 created complement-activating surface domains due to altered polystyrene re156 packaging and configuration. Indeed, alterations in polymer configuration can incite 157 complement through different pathways. ${ }^{34}$

158 We further observed a similar time-dependent complement activation (through 159 measurements of $\mathrm{C} 3 \mathrm{bc}, \mathrm{C} 3 \mathrm{a}, \mathrm{C} 5 \mathrm{a}$ and $\mathrm{sC} 5 \mathrm{~b}-9)$ profile by the particles in human 
160 blood, but unlike pig blood complement activation by all particles were comparable at 161 late time points (Supplementary Fig. 2). The reasons for these differences are not 162 clear, but may be related to differences in protein corona on particles in pig and 163 human blood and subsequent complement activation by adsorbed proteins. ${ }^{35}$ Since 164 these particles did not trigger complement activation instantaneously in porcine and 165 human blood, we next assessed haemodynamic responses on particle injection in pigs. 166 Particles were injected intravenously at an equivalent surface area $(\sim 114,300$ $167 \mathrm{~mm}^{2} / 20 \mathrm{~kg}$ body weight) into pigs, which demonstrated a different trend in 168 cardiopulmonary responses. Immediately on injection, spheres elevated PAP with a 169 concomitant decline in SAP (Fig. 2c \& d). Haemodynamic disturbances, however, 170 were restored within $5 \mathrm{~min}$ of injection. These haemodynamic responses were 171 comparable to a $0.5 \mathrm{mg} / \mathrm{kg}$ zymosan dose (Supplementary Fig. 3), but unlike spheres, 172 zymosan is a potent and an instantaneous activator of the complement system. ${ }^{8,21,22}$ 173 Administration of the prostaglandin inhibitor indomethacin attenuated zymosan- and 174 sphere-induced rises in PAP (Supplemenatry Fig. 3), indicating that particle-induced 175 rises in PAP can at least be partially attributed to prostaglandin release from PIMs. In 176 contrast to spheres, neither rods nor disks induced notable cardiopulmonary 177 disturbances and minute PAP rises were peaked slightly later (Fig. 2c). In all cases, 178 PAP rises were returned to background level by $10 \mathrm{~min}$ and there was no further 179 elevation at 20 min post injection, despite the fact that rods and disks induced notable 180 complement activation in pig blood from 10 min onward. The shape-dependent 181 cardiopulmonary distress differences in pigs were also reflected by the ability of the 182 spheres to elevate thromboxane B2 (TxB2), an inactive metabolite of the 183 vasoconstrictor TxA2 released predominantly by macrophages, at the peak level of 184 PAP (Fig. 2e)..$^{35}$ 

may be complement-independent and could be related to kinetics of particle clearance by PIMs. Accordingly, robust particle removal from the blood (as in spheres or zymosan particles) may initiate cardiopulmonary disturbances. Next, we used

189 rhodamine-labelled particles to compare their clearance rates from the porcine 190 circulation on intravenous injection at an equivalent particle number $\left(1.5 \times 10^{11}\right.$ 191 particles $/ 20 \mathrm{~kg}$ body weight). The results in Fig. 3 show that both rods and disks 192 circulate longer than spheres. Notably, a large proportion of spheres are cleared from 193 the blood within 2 min of injection compared with rods and disks, which coincide 194 with peak PAP and $\mathrm{TxB}_{2}$ levels. These findings corroborate with the suggestion that 195 immediate and robust particle phagocytosis by PIMs may largely control the 196 magnitude of cardiopulmonary responses. ${ }^{15}$ Thus, to further assess a role for PIMs in 197 cardiopulmonary distress responses, we performed a second set of experiments in pigs 198 where the majority of PIMs were depleted by prior administration of clodronate199 encapsulated liposomes (Fig. 4). ${ }^{37}$ Indeed, on PIM depletion, carboxylated sphere 200 (injected at $1.5 \times 10^{11}$ particle $/ 20 \mathrm{~kg}$ body weight)-mediated PAP and $\mathrm{TxB}_{2}$ rises were 201 dramatically dampened. Furthermore, similar observations were obtained on injection 202 of other particle types such as sulfated polystyrene particles (500 $\mathrm{nm}$ in size and at 1.5 $203 \mathrm{x} 10^{11}$ particle/20 kg body weight) and PEGylated doxorubicin-encapsulated 204 liposomes (200 $\mathrm{nm}$ in size and $10 \mathrm{mg}$ total lipid/20 kg body weight) following PIM 205 depletion (Fig. 4). The latter is interesting, since PEGylated liposomes induce rapid 206 complement activation (within minutes) in contact with blood. ${ }^{38}$ Again, this suggest a 207 critical role for PIMs in directing cardiopulmonary responses irrespective of 208 complement activation. We also emphasize that the macrophage depletion strategy 209 with clodronate-encapsulated liposomes is not exclusive to PIMs. ${ }^{39}$ This approach 
210 may have further depleted some circulating monocytes as well as other intravascular

211 macrophages (e.g., spleen marginal zone and red-pulp macrophages) ${ }^{39}$ and therefore

212 we cannot exclude a possible contributing role for these phagocytes in

213 cardiopulmonary distress.

214 We further used radiolabelled particles to investigate their clearance kinetics

215 and biodistribution in the mouse model, which physiologically does not possess PIMs.

216 The results showed similar particle shape-dependent blood clearance profile as in pigs

217 (Supplementary Fig. 4). After 10 min of injection the blood concentration of all three-

218 particle types was similar and corresponded to $<10 \%$ of the administered dose. At this

219 point, biodistribution analysis confirmed comparable levels of particle confinement to

220 the murine macrophage-rich organs (liver and spleen), irrespective of particle shape

221 (Supplementary Fig. 4). This also suggests that deviation from sphericity, and at least

222 with current nanoparticle dimensions, has no significant effect on the overall tissue

223 distribution of nanoparticles. Accordingly, compared with spherical particles, the

224 initial higher blood concentration of rods and disks (i.e., 0-2 min) is a reflection of

225 their lesser localization to the liver and spleen macrophages within this time frame.

226 The intravenous route of administration rapidly exposes particles to the lung 227 capillaries, ${ }^{31}$ thereby placing the particles in direct and immediate contact with 228 pulmonary macrophages in pigs. The dimensions of rods and disks used in this study, 229 however, are not sufficiently large (i.e., they are not in micron-range dimensions) to 230 allow conditions of shear flow and vascular anatomy to modulate particle dynamics 231 and orientation in the systemic circulation. ${ }^{40,41}$ Therefore, it is highly plausible that 232 rods and disks of current dimensions assume random orientation in the blood, where 233 an end-on (for rods) or edge-on (for disks) approach (high curvature domains) may 234 overcome rapid sensing and recognition by macrophages, thereby explaining their 
235 slower clearance rate from the blood compared with spheres. Accordingly, only a

236 fraction of rods and disks are sensed by PIMs at a typical blood circulation round, 237 which correlate with low PAP rises.

238 In agreement with this notion, J774 macrophages under static conditions also 239 showed the trend of significantly faster uptake of spheres compared with rods and 240 disks in the first minute of mixing, followed by comparable uptake levels at all later 241 time points where particles have settled and macrophages have the opportunity to 242 engulf particles of different orientations (Supplementary Fig. 5). The slower clearance 243 rate of rods and disks by PIMs may have therefore triggered a desensitization 244 process $^{42}$ and consequentially prevented the release of secondary mediators 245 responsible for initiating cardiopulmonary distress. Clinical studies have also shown 246 that slowing the infusion rate of nanomedicines decreases the magnitude of adverse 247 reactions in sensitive human subjects. ${ }^{4,5}$ Therefore, a plausible explanation for this 248 phenomenon is the reduced rate of particle presentation to the putative induced 249 pulmonary macrophages in sensitive subjects. ${ }^{15}$ Finally, our results may also explain 250 why administration of a recently designed artificial phospholipid disk-shaped particle 251 did not incite adverse cardiopulmonary distress in pigs, as they may have been cleared 252 from the blood at a slow rate by PIMs. ${ }^{43}$

\section{Particle hitch-hiking on erythrocytes}

255 Earlier, it was shown that particles of different sizes (e.g., 110-1100 nm) and surface

256 functionalities (e.g., carboxyl, amine, aldehyde and polyethylene amine) can adsorb to 257 erythrocytes, which subsequently improve their circulation times. ${ }^{29,30}$ Since PIMs 258 played a central role in injection reactions to particles, we reasoned that a transient 259 delay in extraction of spherical particles by macrophages through erythrocyte 'hitch- 
260 hiking' may dampen haemodynamic disturbances. To test this hypothesis we first

261 used larger $(750 \mathrm{~nm})$ carboxylated polystyrene spheres to induce more efficient

262 complement activation. ${ }^{18,21}$ The results in Fig. 5a-d show association of carboxylated

263 spheres to both human and pig erythrocytes in the absence of plasma, which remain

264 bound upon plasma restoration. The results further demonstrates that spheres in free

265 form or attached to erythrocytes induce comparable complement activation (Fig. 5e).

266 On intravenous injection, erythrocyte 'hitch-hiked' particles did not elevate PAP

267 considerably, but unbound particles induced a substantial rise in PAP (Fig. 5f). The

268 low PAP responses observed with 'hitch-hiked' systems may have been caused by the

269 presence of the $30 \%$ unbound particles (Fig. 5c). These haemodynamic observations

270 were also reproducible with poor complement activating $500 \mathrm{~nm}$ spheres bound to

271 erythrocytes (Supplementary Fig. 6), which additionally highlight detection of

272 increased thromboxane levels on administration of unbound particles as opposed to

273 'hitch-hiked' particles. Taken together, these results imply that erythrocyte 'hitch-

274 hiking' decreases particle-mediated cardiopulmonary distress by avoiding early

275 interactions with macrophages irrespective of complement activation.

276 Finally, we suggest that erythrocyte 'hitch-hiking' may serve as an alternative 277 approach for alleviating the reported adverse injection reaction to currently available 278 spherically-shaped anti-cancer nanomedicines such as poly(cyanoacrylate) and 279 poly(DL-lactide-co-glycolide) nanoparticles carrying cytotoxic agents and their 280 derivatives thereof. ${ }^{1,31,44}$ Indeed, these particles can adhere to erythrocytes in the 281 absence of plasma and remain bound even following plasma restoration. ${ }^{30}$ 


\section{Conclusions}

286 We demonstrated for a set of different-shaped polystyrene particles how robust

287 macrophage clearance dictates the extent of cardiopulmonary responses, irrespective

288 of complement activation. The type of macrophage receptor(s) and associated

289 signalling presumably regulates this transitional link between robust phagocytosis and

290 cardiopulmonary distress. Although, the identity of these receptors remains unknown,

291 we dampened cardiopulmonary distress in pigs by two independent approaches that

292 attenuated rapid particle-macrophage interactions. The first approach was to use

293 particles displaying rod or disk morphologies with dimensions below $500 \mathrm{~nm}$. The

294 second approach resolved adverse injection reaction to spherical particles through

295 their prior adherence to erythrocytes. These strategies avoided the use of

296 immunological or pharmacological manipulations. ${ }^{45-48}$ These 'simple-by-design'

297 approaches may be extended to PRINT technology (Particle Replication in Non-

298 wetting Templates) ${ }^{49}$ for identification of other geometries and particle dimensions

299 for overcoming injection-related reactions to future nanomedicines. Even with

300 spherically shaped particles, erythrocyte "hitch-hiking" may provide a viable clinical

301 solution for nanomedicine administration and salvage the use of currently available

302 polymeric-based drug carriers for different therapeutic interventions. ${ }^{31}$ Finally, we

303 suggest that PIMs act as major players in particle-mediated injection reactions, while

304 the exact role of complement needs to be explored in detail. Although inadvertent

305 complement activation may still play a role in injection reactions, our observations

306 suggest that in vitro complement assessment alone may not be a sufficiently sensitive

307 approach to predict adverse injection reactions and for preselecting patients for safe

308 administration of nanopharmaceuticals.

309 
311 Methods and any associated references are available in the online version of the

312 paper.

\section{References}

315 1. Kattan, J. et al. Phase I clinical trial and pharmacokinetic evaluation of doxorubicin 316 carried by polyisohexylcyanoacrylate nanoparticles. Invest. New Drugs 10, 191-199 (1992).

2. Laing, R. B., Milne, L. J., Leen, C. L., Malcolm, G. P. \& Steers, A. J. Anaphylactic reactions to liposomal amphotericin. Lancet 344, 682 (1994).

3. Uziely, B. et al. Liposomal doxorubicin: antitumor activity and unique toxicities during two complementary phase I studies. J. Clin. Oncol. 13, 1777-1785 (1995).

4. Moghimi, S. M., Wibroe, P. P., Helvig, S. Y., Farhangrazi, Z. S. \& Hunter, A. C. Genomic perspectives in inter-individual adverse responses following nanomedicine administration: The way forward. Adv. Drug Deliver. Rev. 64, 1385-1393 (2012).

5. Szebeni, J. Complement activation-related pseudoallergy: a stress reaction in blood triggered by nanomedicines and biologicals. Mol. Immunol. 61, 163-173, (2014).

6. Kastl, S. P. et al. In human macrophages the complement component C5a induces the expression of oncostatin M via AP-1 activation. Arterioscler. Thromb. Vasc. Biol. 28, 498-503 (2008).

7. Woodruff, T. M., Nandakumar, K. S. \& Tedesco, F. Inhibiting the C5-C5a receptor axis. Mol. Immunol 48, 1631-1642 (2011).

8. Szebeni, J. et al. A porcine model of complement-mediated infusion reactions to drug carrier nanosystems and other medicines. Adv. Drug Deliv. Rev. 64, 1706-1716 (2012).

9. Szebeni, J. et al. Complement activation-related cardiac anaphylaxis in pigs: role of C5a anaphylatoxin and adenosine in liposome-induced abnormalities in ECG and heart function. Am. J. Physiol. Heart Circ. Physiol. 290, H1050-H1058 (2006).

10. Warner, A. E. Pulmonary intravascular macrophages. Role in acute lung injury. Clin. Chest Med. 17, 125-135 (1996).

11. Schneberger, D., Aharonson-Raz, K. \& Singh, B. Pulmonary intravascular macrophages and lung health: what are we missing? Am. J. Physiol. Lung Cell Mol. Physiol. 302, L498-L503 (2012).

12. Longworth, K. E., Westgate, A. M., Grady, M. K., Westcott, J. Y. \& Staub, N. C. Development of pulmonary intravascular macrophage function in newborn lambs. $J$. Appl. Physiol. 73, 2608-2615 (1992).

13. Csukás, D., Urbanics, R., Wéber, G., Rosivall, L. \& Szebeni, J. Pulmonary intravascular macrophages: prime suspects as cellular mediators of porcine CARPA. Eur. J. Nanomed. 7, 27-36 (2015).

14. Zhang, X. et al. Regulation of Toll-like receptor-mediated inflammatory response by complement in vivo. Blood 110, 228-236 (2007).

15. Moghimi, S. M. Complement propriety and conspiracy in nanomedicine: perspective and a hypothesis. Nucleic Acid Ther. 26, 67-72 (2016).

16. Keyes, J. W., Jr., Wilson, G. A. \& Quinonest, J. D. An evaluation of lung uptake of colloid during liver imaging. J. Nucl. Med. 14, 687-691 (1973).

17. Imarisio, J. J. Liver scan showing intense lung uptake in neoplasia and infection. $J$. Nucl. Med. 16, 188-190 (1975). 
18. Moghimi, S. M. et al. Complement activation cascade triggered by PEG-PL engineered nanomedicines and carbon nanotubes: the challenges ahead. J. Control. Release 146, 175-181, (2010).

19. Chanan-Khan, A. et al. Complement activation following first exposure to pegylated liposomal doxorubicin (Doxil): possible role in hypersensitivity reactions. Ann. Oncol. 14, 1430-1437 (2003).

20. Szebeni, J. et al. Liposome-induced complement activation and related cardiopulmonary distress in pigs: factors promoting reactogenicity of Doxil and AmBisome. Nanomedicine 8, 176-184 (2012).

21. Moghimi, S. M., Hamad, I., Andresen, T. L., Jorgensen, K. \& Szebeni, J. Methylation of the phosphate oxygen moiety of phospholipid-methoxy(polyethylene glycol) conjugate prevents PEGylated liposome-mediated complement activation and anaphylatoxin production. FASEB J. 20, 2591-2593 (2006).

22. Andersen, A. J. et al. Single-walled carbon nanotube surface control of complement recognition and activation. ACS Nano 7, 1108-1119 (2013).

23. Moghimi, S. M. \& Murray, J. C. Poloxamer-188 revisited: a potentially valuable immune modulator? J. Natl. Cancer Inst. 88, 766-768 (1996).

24. Laverman, P., Carstens, M. G., Storm, G. \& Moghimi, S. M. Recognition and clearance of methoxypoly(ethyleneglycol)2000-grafted liposomes by macrophages with enhanced phagocytic capacity. Implications in experimental and clinical oncology. Biochim. Biophys. Acta 1526, 227-229 (2001).

25. Kolhar, P. et al. Using shape effects to target antibody-coated nanoparticles to lung and brain endothelium. Proc. Natl. Acad. Sci. U.S.A. 110, 10753-10758, (2013).

26. Lu, Z. S., Qiao, Y., Zheng, X. T., Chan-Park, M. B. \& Li, C. M. Effect of particle shape on phagocytosis of CdTe quantum dot-cystine composites. MedChemComm 1, 84-86 (2010).

27. Champion, J. A. \& Mitragotri, S. Role of target geometry in phagocytosis. Proc. Natl. Acad. Sci. U.S.A. 103, 4930-4934 (2006).

28. Geng, Y. et al. Shape effects of filaments versus spherical particles in flow and drug delivery. Nat. Nanotechnol. 2, 249-255 (2007).

29. Chambers, E. \& Mitragotri, S. Prolonged circulation of large polymeric nanoparticles by non-covalent adsorption on erythrocytes. J. Control. Release 100, 111-119 (2004).

30. Anselmo, A. C. et al. Delivering nanoparticles to lungs while avoiding liver and spleen through adsorption on red blood cells. ACS Nano 7, 11129-11137 (2013).

31. Moghimi, S. M., Hunter, A. C. \& Andresen, T. L. Factors controlling nanoparticle pharmacokinetics: an integrated analysis and perspective. Annu. Rev. Pharmacol. Toxicol. 52, 481-503 (2012).

32. Jansen, J. H., Hogasen, K. \& Mollnes, T. E. Extensive complement activation in hereditary porcine membranoproliferative glomerulonephritis type II (porcine dense deposit disease). Am. J. Pathol. 143, 1356-1365 (1993).

33. Wibroe, P. P., Ahmadvand, D., Oghabian, M. A., Yaghmur, A. \& Moghimi, S. M. An integrated assessment of morphology, size, and complement activation of the PEGylated liposomal doxorubicin products Doxil ${ }^{\circledR}$, Caelyx ${ }^{\circledR}$, DOXOrubicin, and SinaDoxosome. J. Control. Release 221, 1-8 (2016).

34. Hamad, I. et al. Distinct polymer architecture mediates switching of complement activation pathways at the nanosphere-serum interface: implications for stealth nanoparticle engineering. ACS Nano 4, 6629-6638 (2010).

35. Chen, F. et al. Complement proteins bind to nanoparticle protein corona and undergo dynamic exchange in vivo. Nat. Nanotechnol. doi:101038/NNANO.2016.269 (2016)

36. Montalescot, G. et al. Evaluation of thromboxane production and complement activation during myocardial ischemia in patients with angina pectoris. Circulation 84, 2054-2062 (1991). 
37. Gaca, J. G. et al. Prevention of acute lung injury in swine: depletion of pulmonary

\section{Acknowledgements}

446 S.M.M. acknowledges financial support by the Danish Agency for Science,

447 Technology and Innovation (Det Strategiske Forskningsråd), reference 09-065746.

448 T.E.M. acknowledges financial support from the European Community's Seventh

449 Framework Programme under grant agreement number 602699 (DIREKT). S.M.

450 acknowledges support from the National Institutes of Health (R01HL129179). We

451 further acknowledge Nader Payemi (University of Copenhagen) in assisting with

452 scanning electron microscopy studies and Hycult Biotech for providing the pig C5a

\section{ELISA kit.}




\section{Author Contributions}

455 S.M.M. and P.P.W. conceived the idea. P.P.W., A.C.A., P.H.N., A.S., V.G., R.U. and

456 S.M.M. performed experiments. All authors designed, analysed and discussed data.

457 P.P.W. and S.M.M. wrote the paper with contributions from all co-authors. All co458 authors critically revised the manuscript.

459

460 Additional information

461 Supplementary information is available in the online version of the paper. Reprints

462 and permissions information is available online at www.nature.com/reprints.

463 Correspondence and requests for materials should be addressed to S.M.M.

464

465 Competing financial interests

466 The authors declare no competing financial interests.

467 
Figure 1 | Graphical and scanning electron microscopy (SEM) representation of spheres, rods, and disks. a, true relative size and shape with colours representing Gaussian curvature (assuming rods and disks as prolate and oblate spheroids, respectively). b-d, SEM images of spheres (b), rods (c) and disks (d). Scale bars: 500 nm.

Figure 2 | Changes in complement activation in pig blood and pig haemodynamic parameters after exposure to spheres (circles), rods (triangles) and disks (squares). a \& b , time-dependent complement activation in pig whole blood shown as percentage of formed $\mathrm{sC} 5 \mathrm{~b}-9$ and $\mathrm{C} 5 \mathrm{a}$, respectively, relative to a $0.2 \mathrm{mg} / \mathrm{mL}$ zymosan response. Values are given as mean \pm s.d. $(n=3$; $\mathrm{sC} 5 \mathrm{~b}-9$ : $p<0.01$ for spheres and disks at 10 and $30 \mathrm{~min}$, and $p<0.001$ for rods at 10 and 30 min compared with the control/background level; non-paired two-sided $t$-test). Complement activation by particles was compared on an equivalent surface area of $\sim 14,500 \mathrm{~mm}^{2} / \mathrm{mL}$ of blood. Absolute values of complement activation products are presented in Supplementary Fig. 1. c, time-dependent changes in pulmonary arterial pressure (PAP) on particle injection compare with background (resting phase, before $0 \mathrm{~min}$ ). Particles (given on an equivalent surface area of $\sim 114,300 \mathrm{~mm}^{2} / 20 \mathrm{~kg}$ body weight) were injected at zero time. Inset: Integrated area under the curve (AUC) of the changes in PAP during the first 10 min of injection. d, changes in the systemic arterial pressure (SAP) on particle injection compared with background (resting phase, before $0 \mathrm{~min}$ ). e, changes in levels of thromboxane $\mathrm{B} 2$ (TxB2) on particle injection compared with background (resting phase, before $0 \mathrm{~min}$ ). The results from pig experiments are expressed as mean \pm s.e.m. $(\mathrm{n}=3)$.

Figure 3 | Circulation profile of spheres, rods and disks following intravenous injection into pigs. Particles were injected at a dose of $1.5 \times 10^{11}$ particles $/ 20 \mathrm{~kg}$ body weight. Spheres are cleared faster compared to rods and disks. The inset is a magnified representation of early time points. The results are expressed as mean \pm s.e.m ( $\mathrm{n}=3$ ). $p<0.05$ (non-paired two-sided $t$-test) for all points between $30 \mathrm{~s}$ to $3 \mathrm{~min}$, comparing spheres with rods and disks.

Figure 4 | Dampening of particle-mediated haemodynamic changes in pigs following pulmonary intravascular macrophage (PIM) depletion. a, number of positive PIM cells per high-powered field in lung samples from untreated and clodronate-liposome-treated pigs (the results represent 15 random biopsy lung specimens per animal \pm s.e.m.; $n=2$ pigs per group). $\mathbf{b}$, time-dependent changes in pulmonary arterial pressure (PAP) in control and clodronate-liposome-treated pigs. Animals were injected intravenously with spherical carboxylated polystyrene particles of $500 \mathrm{~nm}$ in size $\left(1.5 \times 10^{11}\right.$ particle $/ 20 \mathrm{~kg}$ body weight $)$ at zero time. c, comparison of maximum PAP in control and clodronate-liposometreated pigs on intravenous injection of $500 \mathrm{~nm}$ carboxylated polystyrene particles (C-500nm), $750 \mathrm{~nm}$ sulfated polystyrene particles (S-750nm) and 200 nm PEGylated liposomes (Lip 200nm). Polystyrene particles were injected at a dose of $1.5 \times 10^{11}$ particle $/ 20 \mathrm{~kg}$ body weight and liposomes at a dose of $10 \mathrm{mg}$ total lipid/20 kg body weight. d, changes in levels of thromboxane B2 (TxB2) on particle injection in control and clodronate-liposome-treated pigs. The results in b, c \& $\mathbf{d}$ are mean \pm s.e.m. ( $n=2$ pigs per group). In a, c \& d, open columns 
516 represent control animals (pre-treated with control/blank liposomes) and black 517 columns represent pigs pre-treated with clodronate-encapsulated liposomes, 518 respectively. ${ }^{*} p<0.05$, non-paired two-sided $t$-test.

Figure 5 | Overcoming adverse reactions to spheres through erythrocyte 'hitchhiking'. a, differential interference contrast/fluorescence microscopy images of adhered $750 \mathrm{~nm}$ carboxylated polystyrene particles to human and pig (inset) erythrocytes. Scale bars: $10 \mu \mathrm{m}$. b, SEM image of a human erythrocyte with adhered polystyrene particles. Scale bar: $1 \mu \mathrm{m}$. c \& d, quantitative assessment of the particleerythrocyte interaction by FACS. The results show the fraction of bound particles and cells for human and pig (c) and how particles are distributed on cells (d). Values are expressed as mean \pm s.e.m. from four individual human donors, each in biological duplicates, and one pig donor in triplicate. e, complement responses ( $\mathrm{sC} 5 \mathrm{~b}-9$ measurements) to erythrocyte-bound and unbound particles in human and pig whole blood. In c, d \& e the ratio of human and pig erythrocytes to particles was 2:1 corresponding to $1.13 \times 10^{9}$ and $1.69 \times 10^{9}$ spheres/incubation, respectively. Values are expressed as mean \pm s.d. (see methods for statistical details). f, haemodynamic changes in pigs measured by changes in pulmonary arterial pressure (PAP). Total number of particles injected in both cases was $8.6 \times 10^{9} / 20 \mathrm{~kg}$ body weight. Inset: Area under the curve (AUC) for particles that are bound $(B)$ to erythrocytes or unbound $(U)$. Values are expressed as mean \pm s.e.m. for two pigs.

538 
541 Preparation and characterization of particles. Plain carboxylated polystyrene particles of 200, 500 and $750 \mathrm{~nm}$ and sulfated polystyrene particles $(500 \mathrm{~nm})$ were purchased from Polysciences Inc. (Warrington, PA, USA). For some studies FITC- or rhodamine-labelled carboxylated polystyrene particles were used. The $200 \mathrm{~nm}$ particles were stretched into rods and disks either by a one-dimensional or twodimensional film stretching method, respectively, as previously described..$^{27}$ Briefly, $10^{13}$ polystyrene spheres were first embedded into a hot water soluble polyvinyl alcohol film $(10 \% \mathrm{w} / \mathrm{v}$ in water) with $2 \%(\mathrm{w} / \mathrm{v})$ glycerol. Films were then mounted and mechanically stretched in either one or two dimensions in oil at $120^{\circ} \mathrm{C}$. Films were then dissolved in $70^{\circ} \mathrm{C}$ water for $2 \mathrm{~h}$ and then centrifuged at $8,000 \mathrm{~g}$ to isolate the particles. Particle suspensions were centrifuged in water 10 more times and finally passed through a $170 \mu \mathrm{m}$ filter. Scanning electron micrographs were taken on an FEI XL40 and imaged at $5-10 \mathrm{kV}$ acceleration voltage at $5 \mathrm{~mm}$ working distance.

PEGylated liposomes (100 and $200 \mathrm{~nm}$, respectively) resembling Doxil ${ }^{\circledR}$ in lipid composition and doxorubicin content were prepared as described before. ${ }^{33}$ Size analysis was performed by Nanoparticle Tracking Analysis following sample dilution $\left(\mathrm{x} 10^{6}\right)$ with $10 \mathrm{mM} \mathrm{NaCl}$ and monitored with an LM20 NanoSight mounted with a blue $(405 \mathrm{~nm})$ laser (Malvern Instruments, UK) using the Nanosight 2.3 software for data analysis. ${ }^{33}$

In vitro complement activation in whole blood. For activation of the complement system, blood was drawn from healthy human subjects according to local approved protocols and individual consent into blood tubes containing the anticoagulant lepirudin (Refludan ${ }^{\circledR}$, Hoechst, Frankfurt am main, Germany), which does not affect complement system. $^{50}$ Pig blood was also collected in lepirudin blood tubes. Measurements on human whole blood (WB) were based on three individual donors. Measurements on pig WB were done in three different experiments using blood from a healthy pig. Particle concentration was normalized to yield constant exposed surface area. Briefly, $20 \mu \mathrm{L}$ particles, PBS or zymosan $(0.2 \mathrm{mg} / \mathrm{mL})$ were added to $\mathrm{WB}$ corresponding to a volume of $\sim 80 \mu \mathrm{L}$ plasma (i.e $160 \mu \mathrm{L}$ human $\mathrm{WB}$ and $120 \mu \mathrm{L}$ pig WB) and incubated at $37^{\circ} \mathrm{C}$ for a range of time points $(1-30 \mathrm{~min})$ followed by dilution in cold diluent containing EDTA to stop complement activation. After centrifugation, human $\mathrm{C} 3 \mathrm{bc}$ and $\mathrm{sC} 5 \mathrm{~b}-9$ was quantified by ELISA as described elsewhere. ${ }^{51} \mathrm{Pig}$ sC5b-9 determination was done as described earlier. ${ }^{32}$ Human and pig C5a was quantified using commercial available kits (Hycult, Uden, the Netherlands). For particles bound to erythrocytes, the blood was pretreated as stated below.

Interaction between C3a and particles. In addition to the complement markers above, C3a was also included to monitor complement activation. However, due to a reduced level of measured $\mathrm{C} 3 \mathrm{a}$ in blood when particles were present, a potential interaction between $\mathrm{C} 3 \mathrm{a}$ and particles was investigated. Purified human C3a (Hycult, Uden, The Netherlands) was mixed with a pool of human EDTA-treated plasma from 9 donors, reaching final C3a concentrations of $0-5400 \mathrm{ng} / \mathrm{mL}$. This concentration range was selected to mimic the concentrations reached in zymosan-induced in vitro complement responses. Accordingly, the three particle shapes were introduced in amounts mimicking the incubations for complement activation in the blood. After 30 min incubation at $37^{\circ} \mathrm{C}$, particles were pelleted and the concentration of $\mathrm{C} 3 \mathrm{a}$ in the supernatant was measured by ELISA (Hycult, Uden, the Netherlands) and compared with plasma samples incubated without particles. 
591 Haemodynamic measurements in pigs. In vivo studies were performed on Yorkshire 592 pigs $(23-27 \mathrm{~kg})$. This method has previously been fully detailed and validated, and approved by Semmelweis University Animal Subject Review Committee. ${ }^{8,9,45}$ Briefly, each pig was randomly selected and initially sedated with $40 \mathrm{mg} / \mathrm{kg}$ ketamine and then anesthetized with sodium pentobarbital (25-150 mg/h). A catheter was advanced into the right jugular vein and into the pulmonary artery for measurement of PAP and SAP. A second catheter was placed through the right femoral artery into the distal aorta, measuring systemic arterial pressure. Electrocardiogram and respiratory output was also continuously recorded and blood samples were drawn through the left jugular vein before and following particle injection to monitor blood markers. For particles bound to erythrocytes, blood samples were initially drawn into lepirudin tubes and used for erythrocyte isolation. Particles were administered through the left jugular vein in a blind manner. For treatment with indomethacin, PBS was slowly added to a solution of $6.5 \mathrm{mg} / \mathrm{mL}$ in ethanol, to reach a final indomethacin concentration of $2.6 \mathrm{mg} / \mathrm{mL}$ in $40 \%$ ethanol. A total dose of $1 \mathrm{mg} / \mathrm{kg}$ was slowly administered to the pigs 10-15 min before particle injection. In some experiments, particles were injected $24 \mathrm{~h}$ after PIM depletion. The latter was achieved with 4 intravenous infusions of clodronate-encapsulated multi-lamellar egg phosphatidylcholine/cholesterol (mole ratio 7:3) liposomes of 800-1700 nm range (corresponding to $1.0 \mathrm{~g}$ clodronate $/ 10 \mathrm{~kg}$ body weight) once every $12 \mathrm{~h} .{ }^{37}$ Control animals received an equal volume of empty liposomes. Fifteen random biopsy lung specimens per animal ( $\mathrm{n}=2$ pigs per group) were selected for assessment of PIMs stained by Monastral blue (injected intravenously at a dose of $5 \mathrm{mg} / \mathrm{kg}$ in saline $1 \mathrm{~h}$ before particle administration). ${ }^{37}$ Slides, after fixation in $10 \%$ buffered formalin and subsequent dehydration and paraffin embedding were sectioned into 4-5 $\mu \mathrm{M}$ thickness and then de-parrafinized, rehydrated and stained with eosin. Finally, slides were analysed for the number of positive PIM cells per high-powered field.

Thromboxane B2 was measured from the extracted blood plasma samples using a commercial thromboxane B2 Express EIA kit (Cayman Chemical, Ann Arbor, MI, USA).

Attachment and characterization of particles to erythrocytes. Freshly drawn WB samples were centrifuged at 1,200 $\mathrm{g}$ for $7 \mathrm{~min}$ to pellet cells. Plasma was removed and stored for later use, and buffy coat was discarded. Erythrocytes were then washed in PBS 3 times. No haemolysis was observed during the handling. The final erythrocyte number was counted (Tali Image Cytometer, ThermoFisher Scientific, UK) and mixed with particles dispersed in PBS in an erythrocyte:particle ratio of $\sim 2: 1$ which was found to be optimal, and incubated $37^{\circ} \mathrm{C}$ for $30 \mathrm{~min}$ to allow particle adhesion. Lepirudin-anticoagulated plasma was then added enabling complement activation to occur, following the procedure described above. For preparations with unbound particles, erythrocytes were first incubated in presence of PBS and then reconstituted with lepirudin-anticoagulated plasma prior to mixing with particles for complement activation studies. When particles were absent, PBS was used to achieve constant volume. Erythrocyte samples with bound particles also contained some unbound particles. and quantified by FACS. For FACS characterization, reacted complement samples were diluted in PBS to a total erythrocyte dilution of $10^{4}$ and monitored on a BD FACSArray flow cytometer (BD Biosciences, CA, USA) with flow rate of $0.5 \mu \mathrm{L} / \mathrm{s}$ and side scatter threshold of 3,000 . 
For scanning electron microscopy (SEM), samples were diluted with 4\% formaldehyde and left at room temperature overnight. Thereafter a small volume was

In vivo circulation and biodistribution studies. The blood clearance of rhodaminelabelled particles (spheres, rods and disks) was monitored after a single intravenous injection (1.5 x $10^{11}$ particles $/ 25 \mathrm{~kg}$ body weight) into pigs $(\mathrm{n}=2)$ in a non-blind manner. At selected time points blood samples were removed and analysed for the presence of particles. The blood concentration of particles was estimated from blood samples containing known quantities of labelled particles.

In some experiments, spheres, rods and disks were radiolabelled with ${ }^{3} \mathrm{H}$-oleic acid (Moravek Biochemicals) for biodistribution studies in mice in a non-blind manner. Briefly, $20 \% \mathrm{w} / \mathrm{v}$ particle suspension in water was added to a solution containing $\left.100 \mu \mathrm{L} \mathrm{[}{ }^{3} \mathrm{H}\right]$-oleic acid, $100 \mu \mathrm{L}$ ethanol, and $25 \mu \mathrm{L}$ tetrahydrofuran for 30 min with constant rotation. Particles were washed ten times at $15,000 \mathrm{~g}$ for $30 \mathrm{~min}$ by centrifugation to remove unincorporated tritium and then re-suspended in saline prior to injection. For circulation and biodistribution studies, $5 \times 10^{9}$ radiolabelled particles were injected into tail vein of randomly grouped healthy female BALB/c mice (18-20 g). At specified time points, blood was drawn and mice were sacrificed by asphyxiation. Known weights of blood, liver, spleen, kidney, heart, lungs, brain, and skin were harvested and dissolved overnight at $60{ }^{\circ} \mathrm{C}$ in $5 \mathrm{~mL}$ of Solvable (Perkin Elmer, UT, USA). The next day, Ultima Gold (Perkin Elmer, UT, USA) was added to dissolved organ samples and $\left[{ }^{3} \mathrm{H}\right]$ content was measured using a TriCarb 2100TR scintillation counter. All mouse protocols were approved by the Institutional Animal Care and Use Committee (IACUC) at the University of California at Santa Barbara (CA, USA).

In vitro macrophage uptake. Radiolabelled particles of different shapes were prepared as described above. J774A.1 macrophages (ATCC ${ }^{\circledR}$ TIB-67TM) (American Type Culture Collection, VA, USA) were cultured in standard cell culture conditions $\left(37^{\circ} \mathrm{C}\right.$ in $\left.5 \% \mathrm{CO}_{2}\right)$ in high glucose Dulbecco's Modified Eagle's Medium, DMEM (ATCC, VA, USA), 10\% (v/v) foetal bovine serum, and 1\% penicillin/streptomycin. $1.5 \times 10^{4}$ J774A. 1 cells per well were seeded for $24 \mathrm{~h}$ in a 96 -well plate. Prior to the experiment, particles were resuspended in DMEM containing $20 \%(\mathrm{v} / \mathrm{v})$ fresh BALB/c serum, at $0.1 \mathrm{mg} / \mathrm{mL}$ and introduced to plated J774A.1 cells following removal of DMEM containing FBS and penicillin/streptomycin and 3 washes with PBS. At specified time points cells were washed $3 \mathrm{x}$ with PBS to remove unbound or non-internalized particles. Cells were immediately incubated at $60^{\circ} \mathrm{C}$ for $1 \mathrm{~h}$ in $5 \mathrm{~mL}$ of Solvable and then analysed (as described above) for $\left[{ }^{3} \mathrm{H}\right]$ content. 
Data availability statement

690 All relevant data are available from the authors and/or are included with the 691 manuscript as source data (Fig. 1-5) or supplementary information (Supplementary Fig. 1-6). There are no restrictions on availability.

\section{References}

50. Mollnes, T. E. et al. Essential role of the C5a receptor in E-coli-induced oxidative burst and phagocytosis revealed by a novel lepirudin-based human whole blood model of inflammation. Blood 100, 1869-1877 (2002).

700

51. Bergseth, G. et al. An international serum standard for application in assays to detect human complement activation products. Mol. Immunol. 56, 232-239 (2013).

701 


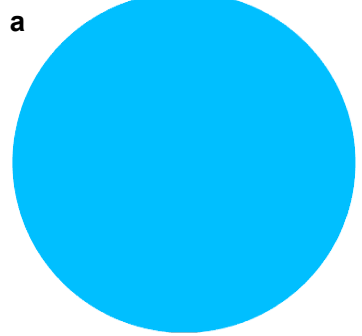

Sphere

$\mathrm{D}=500 \mathrm{~nm}$

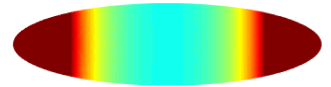

Rod

$D_{1} \times D_{2}=450 \times 120 \mathrm{~nm}$

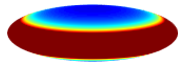

Disk

$D_{1} \times D_{2}=250 \times 75 \mathrm{~nm}$

Low

High

curvature

curvature

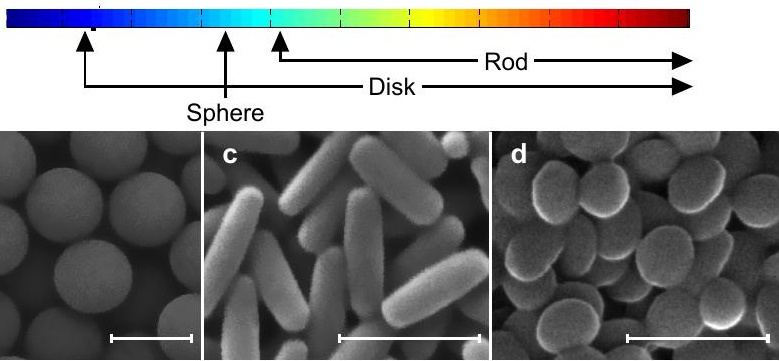



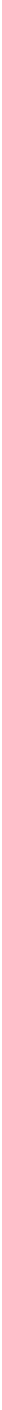


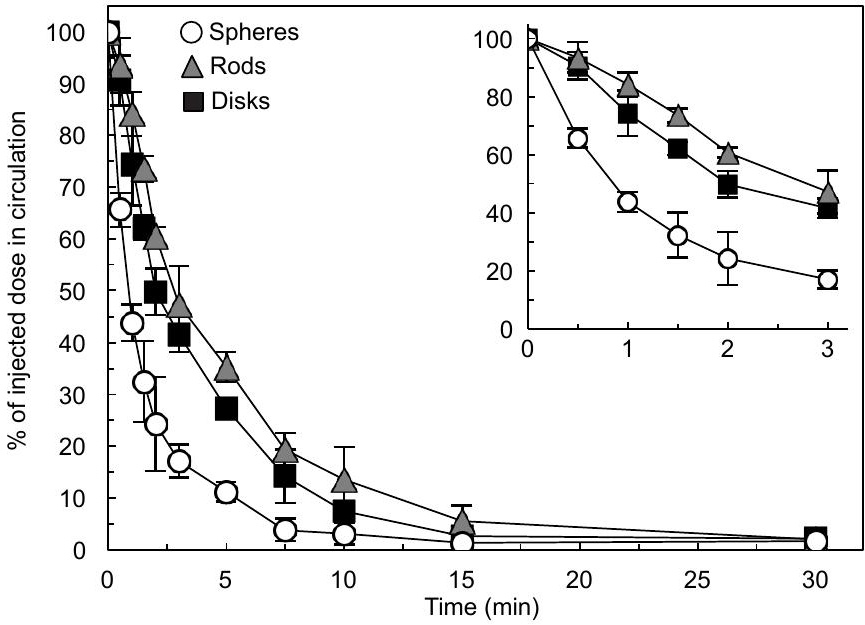


